\title{
Clinical study on surgical treatment of granulomatous lobular mastitis
}

\author{
Chaojie Zhang ${ }^{1}$, Shanshan Lei ${ }^{1}$, Cheng Kong ${ }^{1}$, Yuanzhen Tan ${ }^{2}$, Xu Dai ${ }^{1}$, Jie He ${ }^{1}$, Qiang Xiao ${ }^{3}$, Shulin Huang ${ }^{1}$ \\ ${ }^{1}$ Department of Breast and Thyroid Surgery, ${ }^{2}$ Teaching Affairs Office, Hunan Provincial People's Hospital/The First Affiliated Hospital of Hunan \\ Normal University, Changsha 410005, China; ${ }^{3}$ Department of Burn and Plastic Surgery, Xiangtan Central Hospital, Xiangtan 411100, China \\ Contributions: (I) Conception and design: C Zhang; (II) Administrative support: C Zhang; (III) Provision of study materials or patients: X Dai, J He, \\ S Huang; (IV) Collection and assembly of data: S Lei, C Kong; (V) Data analysis and interpretation: Q Xiao, Y Tan; (VI) Manuscript writing: All \\ authors; (VII) Final approval of manuscript: All authors. \\ Correspondence to: Chaojie Zhang. Department of Breast and Thyroid Surgery, Hunan Provincial People's Hospital/The First Affiliated Hospital of \\ Hunan Normal University, Changsha 410005, Hunan, China. Email: zhangchaojie74@126.com.
}

Background: The etiology and pathogenesis of granulomatous lobular mastitis (GLM) remain unknown, with no unified evaluation criteria or standard treatments. This study aimed to assess the etiology and features of GLM, as well as the effects of surgery (lesion excision + stage I breast reconstruction; LE + BR) for GLM.

Methods: This study evaluated 178 female GLM patients retrospectively in 2006-2015. The surgery and non-surgery groups included 164 and 14 patients, respectively. All patients received conservative therapy (traditional Chinese medicine combined with regional wet compress and pus drainage). In addition, the surgery group $(\mathrm{n}=164)$ underwent LE + BR. Clinical data, including disease course, causes, lesion size, marital status, and treatment approaches, were assessed.

Results: Follow-up was $13-117$ months. Seventy-five of the 178 patients had no overt causes (42.1\%); meanwhile, $63(35.4 \%)$ and $16(9.0 \%)$ had congenital nipple retraction and a history of psychotropic drugs for $>1$ year, respectively. The surgery group showed lesions significantly shrunk ( $\leq 1$ quadrant) with acute inflammation fully controlled; 8 showed recurrence, indicating a cure rate of $95.1 \%(156 / 164)$. In the nonsurgery group, 4 cases showed relapse after $6-14$ months (cure rate $=71.4 \% ; 10 / 14$ ). Therefore, surgical treatment was significantly more efficient than non-surgical treatment $(\mathrm{P}=0.001)$. Kaplan-Meier survival curves for the two treatment types showed a significant difference in recurrence $(\log \operatorname{rank}=11.84, \mathrm{P}<0.001)$.

Conclusions: In GLM patients, LE + BR is safe and effective with respect to cosmetic results, recovery time, and recurrence. Successful surgery should be performed for patients whose lesions $\leq 1$ quadrant, aim to achieve optimal GLM treatment.

Keywords: Conservative therapy; granulomatous lobular mastitis (GLM); intraglandular flap; predisposing factors; surgery

Submitted Jun 14, 2019. Accepted for publication Oct 08, 2019.

doi: 10.21037 /gs.2019.11.12

View this article at: http://dx.doi.org/10.21037/gs.2019.11.12

\section{Introduction}

Granulomatous lobular mastitis (GLM), also known as idiopathic granulomatous mastitis (IGM), is a chronic disease of the breast characterized by lobular necrosis and non-caseified granulomatous inflammation on histological sections after the infectious causes and non-infectious causes have been excluded. The incidence of GLM increased rapidly in recent years (1), but its etiology and pathogenesis is still unknown (2). When looking through the literature (3-6), the researchers have found the main therapies of GLM including surgery, medication, combination of medication and surgery. The medicine regimens comprise 
mainly corticosteroid therapy, corticosteroid combined with immunosuppressants, antibiotics alone, anti-TB treatment, traditional Chinese medicine alone and a combination of Chinese and Western medicine (7).

Since year of 2006, the first case of the study on GLM, our team came to know about the clinical features of the disease and gained some experience on its diagnosis and treatment. On basis of literature and our own clinical experience, we have gradually identified ' $\mathrm{LE}+\mathrm{BR}$ ' as the main therapy approach for GLM, by which satisfactory clinical results have been obtained and reported as follows.

\section{Methods}

\section{Clinical materials}

This study included 219 patients diagnosed with GLM from July 2006 to July 2015. One hundred and seventyeighth patients had complete follow-up data with a deadline of July, 2016. Follow-up datas included: course, lesion size, marriage, place of residence, therapy approaches, possible causes such as nipple retraction, the recurrence of disease and time to disease recurrence. Based on biopsy and culture of the push for pathogen, all patients had pathologic confirmation of GLM and got an exclusion of diagnosis of plasma cell mastitis, Zuska disease, granulomas caused by other diseases like tuberculosis granulomas, Burke sarcoid (sarcoidosis), fungal infection, Wegener granulomas, fat necrosis and breast cancer $(1,8,9)$. The histopathologic results were confirmed by 2 pathologists. All patients had a breast high-frequency color ultrasonography (RS80A, Samsung, Korea) and routine MRI (Magnetom, Trio A Tim; Siemens, Germany) and enhanced scans at same time at first visit. If patients decided to undergo the surgery of lesion excision, she would take USG and MRI again preoperative to assess the range of lesion.

Ethical clearance was obtained from the Hunan Provincial People's Hospital Medical Faculty ethics committee.

\section{Treatment procedures}

All patients underwent two main different treatments respectively depending on the range of breast lesion and lesion within abscess or not. (I) For patients with abscesses in mammary lesion: we took a three-step treatment: firstly, patients had pus drainages by usage of Mammotome or an open surgery, while negative-pressure drainage tube may be placed and biopsy for pathological diagnosis could also be done simultaneously. Secondly, patients took the conservative therapy, including medication therapy and regional wet compress on the lesion. The main drugs of the medication therapy included: the Xihuang capsules-a Chinese traditional medicine (trade name, Xihuangjiaonang; $1.2 \mathrm{~g}, 2$ times a day) and intravenous antibiotics (Levofloxacin Hydrochloride and Sodium Chloride injection, $0.3 \mathrm{~g} 2$ times a day for 1 week). Some patients may add glucocorticoids (methylprednisolone tablets, $8 \mathrm{mg}$, 2 times a day for 2 weeks; then $4 \mathrm{mg}$, 2 times a day for 1 week; and then reduce dosage by half till $4 \mathrm{mg}$ once a day) if she had erythema on lower extremities and was suspicious of having autoimmune disorders. Regional wet compress used $50 \%$ magnesium sulfate, 20 minutes each time, totally over 6 hours a day. Thirdly, when size of the lesion had a significant shrink ( $\leq 1$ quadrant) and the skin swelling became detumescent, the patients could choose an open surgery for lesion excision and breast reconstruction (LE + BR). The essentials of the surgery included: to remove all the breast lesion involving necrotic adipose tissue and mammary glands, the range of which displayed by MRI; to excise lactiferous ducts posterior of nipple which had relationship to the lesion; to reconstruction the breast by transposition with the residual glands, the surrounding tissues such as adipose and fascia tissues from armpit or lateral chest wall or the areas under the folds of the breast, trying to maintain good shape of the breast. Similarly, breast reconstruction may integrate plastic surgery techniques with intraglandular flap, fascia flap or epidermis removal following the excision of skin ulcerations and necrosis. (II) For patients without abscesses in breast lesion: if the range of lesion was larger than two quadrants of ipsilateral breast, the patients would underwent the Xihuang capsules/antibiotics/regional wet compress regimen before lesion excision; if the original lesion was smaller than one quadrant, LE+BR can be done immediately after the short-time medication therapy. Intraglandular flap was enough for cosmic result only. For a small number of patients who refused to the surgery of lesion excision, she would continue the conservative therapy including Xihuang capsules/regional wet compress +/- pus drainage+/- glucocorticoid.

All patients underwent examinations on follow-up visit, including physical palpation and ultrasonography-at a frequency of 1, 3, 6 months, 1 year respectively after surgery and annually after 1 year. Some patients should take another breast MRI scanning if the diagnosis of ultrasonography was uncertain. 
Table 1 Comparative analysis in composition ratio of associated findings in granulomatous lobular mastitis

\begin{tabular}{lcc}
\hline Associated findings & No. of patients $(\mathrm{n}=178)$ & $\%$ \\
\hline Unknown causes & 75 & 42.1 \\
Nipple retraction & 63 & 35.4 \\
Antipsychotic drugs & 16 & 9.0 \\
No breastfeeding & 10 & 5.6 \\
Steroid hormones & 8 & 4.5 \\
Hyperprolactinemia & 2 & 1.1 \\
Trauma & 4 & 2.2 \\
\hline
\end{tabular}

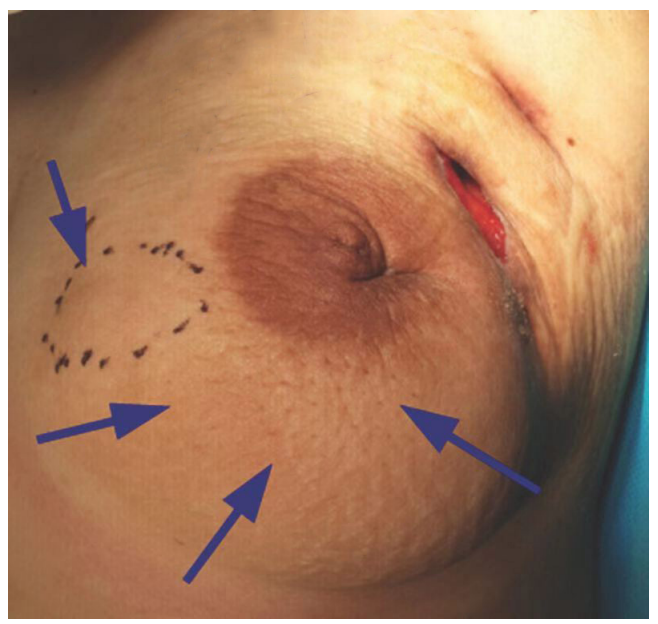

Figure 1 Clinical appearance of a patient with GLM including skin ulcerations, abscesses and fistulae at her first visit at our hospital. GLM, granulomatous lobular mastitis.

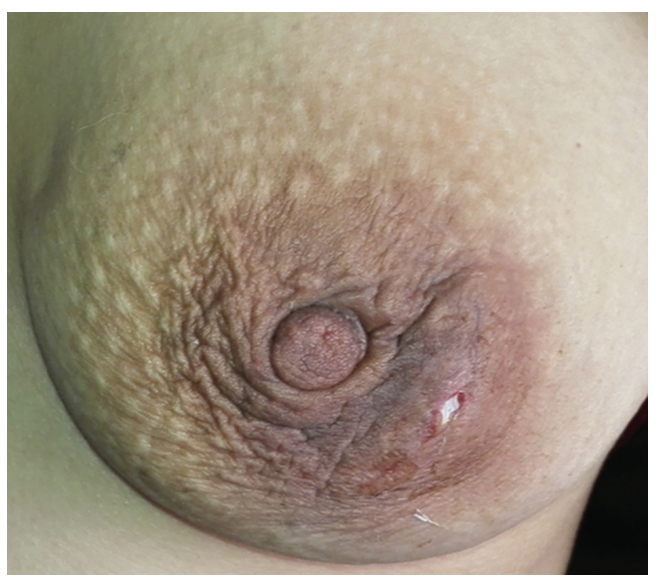

Figure 2 Clinical appearance of a patient with GLM including skin abscesses. GLM, granulomatous lobular mastitis.

\section{Efficacy evaluation}

In this study, a cure standard for GLM was defined by our own clinical observations, because international criterion is unified $(1,4)$. In follow-up period, negative palpation on the ipsilateral breast and no new lesion revealed by USG and by MRI for more than 1 year after surgery therapy or conservative therapy is recognized as criterion of cure. A recurrence on the ipsilateral breast within 1 year after therapy is defined as treatment failure, while majorities of clinical units recognized a recurrence within 6 months as failure in China before (10).

\section{Statistical analysis}

The SPSS 11.5 statistical software package was used for statistical analysis. The cross-table $\chi^{2}$ test was used for a rate comparison. Likelihood Ratio was used for the ratio not met the requirements of fourfold table. Correlation coefficients and P value of Spearman's rho of Nonparametric Correlations were used for correlations analysis. Log Rank of Kaplan-Meier survival curve was tested the recurrence risk during follow-up. Two-tailed probability $\alpha=0.05$ served as the statistical standard of significant difference.

\section{Results}

\section{Predisposing factors of GLM}

One hundred seventy-eight patients (18-58 years, median age, 30 years) presented complete follow-up data in 219 women histologically proven with GLM, accounting for $81.3 \%$. In this study, 75 of 178 patients had no special causes or factors available, accounting for $41.2 \% ; 63$ patients complained of congenital nipple restraction, accounting for $35.4 \%$; 16 patients $(9.0 \%)$ had a long-term use of antidepressants or other antipsychotic drugs for more than 1 year; 10 patients $(5.6 \%)$ did not breastfeed their babies after delivering, 8 of which were never pregnant before; 8 patients (4.5\%) had long-term use of contraceptives more than 2 years; 4 patients $(2.2 \%)$ had a history of breast injuries; only two patients suffered from hyperprolactinemia, accounting for $1.1 \%$ (Table 1).

\section{Clinical manifestations}

All of 178 patients had breast mass with unclear boundaries, ranging from $2 \sim 14 \mathrm{~cm}$ in diameter. Of them, 134 patients $(75.3 \%)$ complained of skin ulcerations and swelling (Figure 1), 127 (71.3\%) with abscesses (Figure 2), 


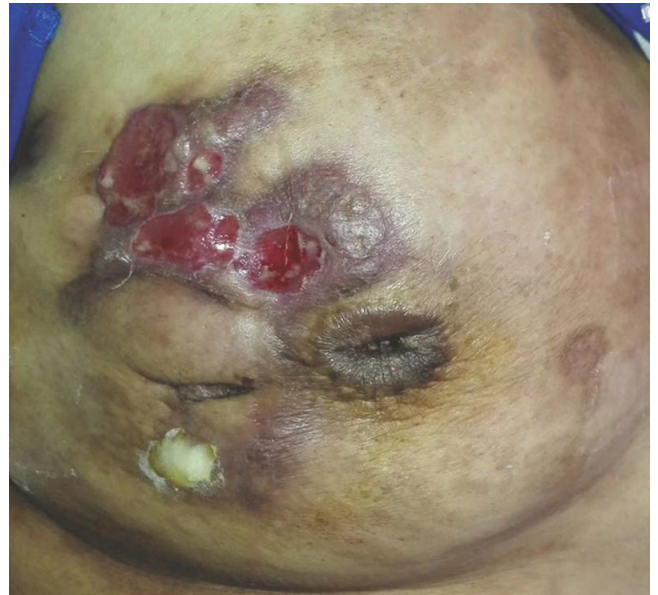

Figure 3 Clinical appearance of a patient with GLM including peau d'orange sign $(\uparrow)$. GLM, granulomatous lobular mastitis.

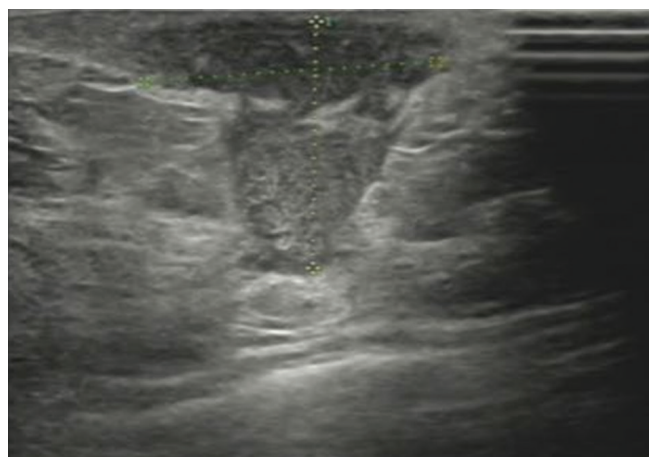

Figure 4 Funnel sign (a hypoechoic, heterogeneous lesion which looks like a pipe with a wide, conical mouth and a narrow stem) is seen in the right breast on ultrasonography in a patient with GLM. GLM, granulomatous lobular mastitis.

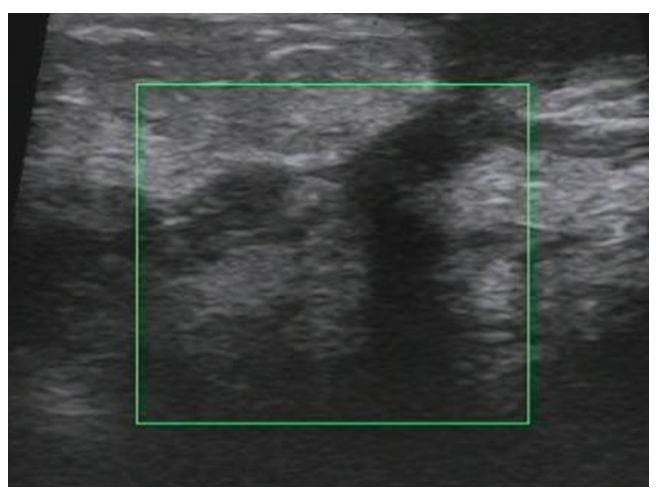

Figure 5 Tunnel sign (a hypoechoic lesion which looks like a subcutaneous passway with exit to the skin) in the left breast on ultrasonography in a patient with GLM. GLM, granulomatous lobular mastitis.

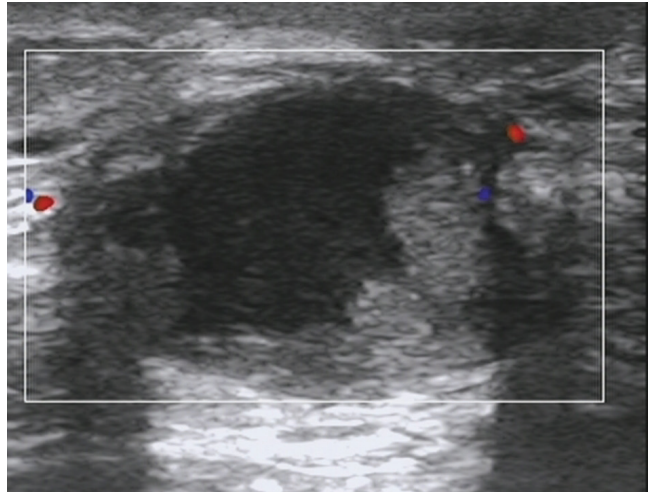

Figure 6 Quicksand sign (a hypoechoic, heterogeneous mass which looks like some fine sand lying at the bottom) is seen in the left breast on ultrasonography in a patient with GLM. GLM, granulomatous lobular mastitis.

$18(10.1 \%)$ with peau d'orange sign, which was easily confused with breast cancer (Figure 3), 6 (3.4\%) with skin erythema on legs.

The duration of GLM which was calculated from the onset to the first visit to our hospital, changed between 3 days to 4 months, mean 1.5 months. The patient with the longest duration experienced the recurrent cycle for several times-the recurrence of the new lesion, pus drainage by open surgery and remission of GLM. Her longest remission period lasted only 8 months. Thirty-seven patients (20.8\%) visited our department after the onset of GLM, 94 patients (52.8\%) after a pus drainage by puncture of Mammotome or open surgery, 47 patients (26.4\%) after painful experience with more than two failed operations.

USG showed ill-defined, hypoechoic, heterogeneous lesions in most of the patients with GLM. Sometimes the breast lesion had specific signs including 'Funnel sign', 'tunnel sign' or 'quicksand sign', while it complicated with abscesses (Figures 4-6). According to the previous experience $(5,11), 26$ patients underwent mammography in our early study, which did not show characteristic signs of the lesion. In late study, patients did not had mammography as a routine examination because of its limitations such as the aggravation of the pain after mammography.

In our study, all of patients had routine examination for pathogen. No microorganisms were demonstrated by Gram stain, and bacterial, fungal and tuberculosis cultures were also negative. All patients got pathological diagnosis by biopsy or open surgery. In late study, a biopsy combined with pus drainage through small incisions were 

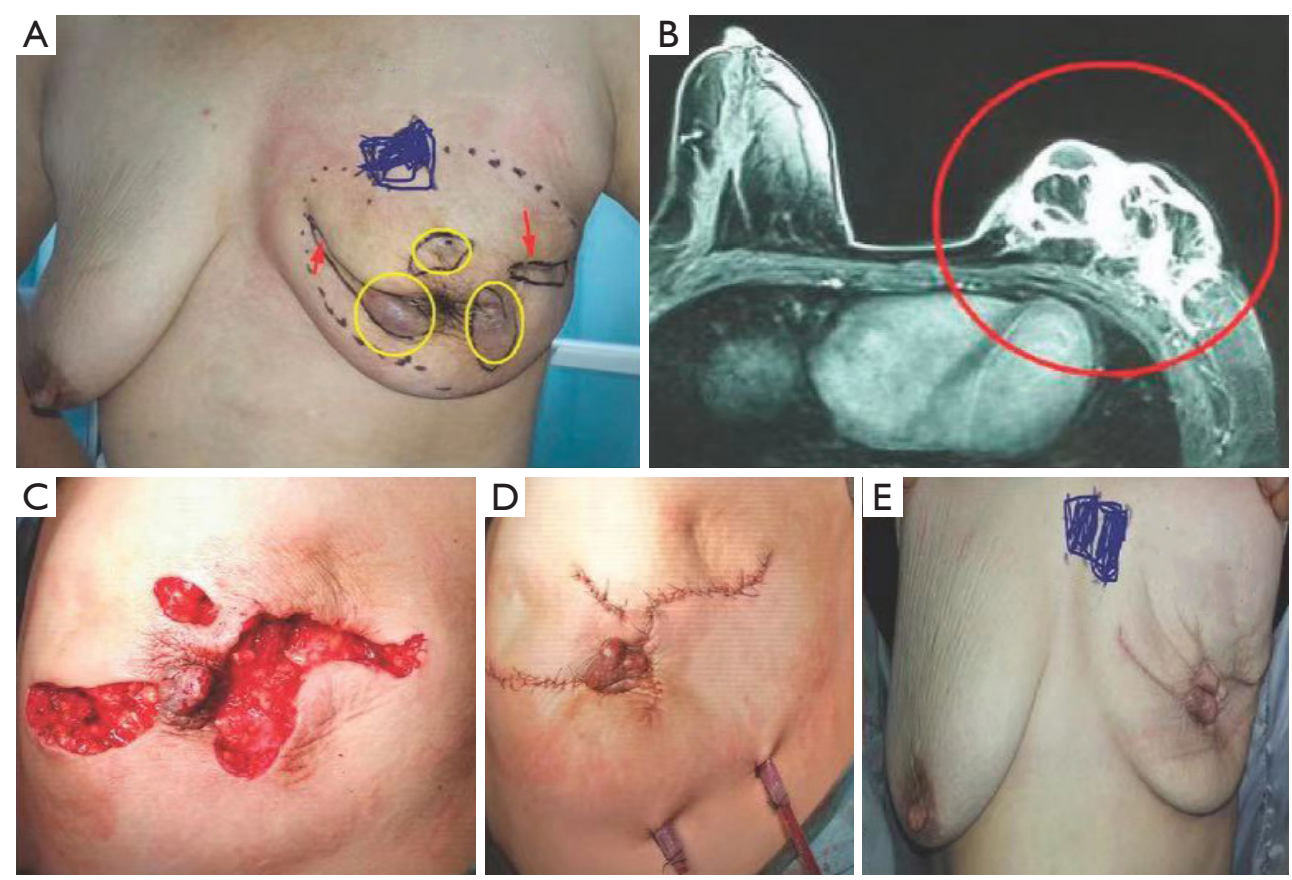

Figure 7 Case 1. A 26-year-old women with a left GLM after the surgery of lesion ( $>3$ quadrants) excision combined with stage I breast reconstruction with intraglandular flap and fascia flap (LE + BR). (A) Preoperatively, after pus drainages for second times at another clinics; (B) images on MRI showed a whole breast lesion of GLM; (C) in the surgery of lesion excision, nipple areola complex had good blood supply; (D) 2 days after the surgery of stage I breast reconstruction with intraglandular flap and fascia flap; (E) 3 months after the surgery. GLM, granulomatous lobular mastitis.

recommended in patients with abscesses, and vacuumassisted breast biopsy were preferred in the patients without abscesses.

\section{Treatment of GLM}

One hundred and sixty-four patients of 178 (164/178) were treated with a comprehensive treatment including surgical therapy for lesion excision and conservative therapy, called surgery group, accounting for $92.1 \%$. They all carried surgical approach of lesion excision combined with stage I breast reconstruction using intraglandular flap and fascia flap ( $\mathrm{LE}+\mathrm{BR}$ ), and the conservative therapy-Xihuang capsules/antibiotics/regional wet compress regimen-before surgery (Figures 7,8). The median duration preoperative was 6 weeks (ranging from 7 days to 4 months). Fourteen (14/178) patients underwent conservative therapy only, called non-surgical group, accounting for $7.9 \%$.

The conservative therapy included traditional Chinese medicine + regional wet compress +/- pus drainage +/glucocorticoids, which lasted to the end of the study, only two cases received glucocorticoids. They took methylprednisolone tablets, $8 \mathrm{mg}, 2$ times a day for 2 weeks at beginning, then reduced dosage by half every week till 4 $\mathrm{mg}$ every day, and last withdraw the tablets. The shortest duration of glucocorticoids therapy was 3 months, the longest was 6 months. The treatment on these two cases was effective except for one case has relapse. None patient received single therapy like antibiotics, glucocorticoids or anti-tuberculosis treatment alone (Table 2). All cases adhered to regional wet compress with $50 \%$ magnesium sulfate.

\section{Efficacy and follow-up results of surgery therapy for GLM}

In 178 cases, follow-up visits by consultations lasted for 13 to 117 months, with median of 29 months. In surgery group, eight patients of 164 had relapse of GLM, the cure rate was $95.1 \%$ (156/164). In non-surgery group, 14 patients underwent the conservative therapy from 6 to 14 months before clinical cure was obtained, with an median of 8.6 months, and four patients of which got lesion recurred, the cure rate $71.4 \%$ (10/14). The clinical 
cure rate of surgery group was significantly higher than that of non-surgery group, with a significant difference of $\chi^{2}=11.517, \mathrm{P}=0.001$. The difference between the two groups was statistically significant by further Spearman's correlation analysis, $\mathrm{r}=-0.254, \mathrm{P}=0.001$ (Table 3). The Kaplan-Meier survival curve of the two types of treatment showed that the relapse of GLM was rarely occurred in the patients undergoing surgical therapy more than 1 year, log rank $=11.84, \mathrm{P}=0.0006$ (Figure 9). The observation of the recurrence cases was not continue in our study.

\section{Discussion}

GLM is a benign and chronic disease of breast. It generally involves the women of childbearing age with a history of birth and breastfeeding. The main complications of GLM include skin ulcerations, the formation of micro abscess or sinuses in regional lesion. In the year of 1972, Kessler and Wolloch (12) firstly reported about GLM, and people

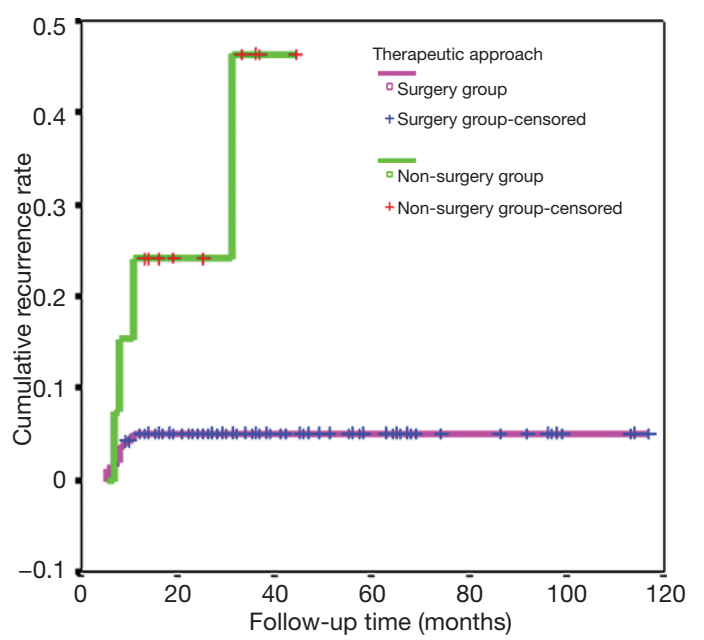

Figure 8 Kaplan-Meier survival curves for recurrence in the two treatment groups. has come to be familiar with the disease. Nowadays, the treatment of GLM still remains controversial, because the etiology and pathogenesis of GLM remains unclear yet. Surgery intervention, antibiotics and corticosteroid therapy are still the three major treatments in different countries, regions and treatment centers (12).

On basis of the clinical features and imaging tests, GLM cases are frequently misdiagnosed to breast cancer because of the diversities of its clinical manifestations, although experienced physicians are prone to make diagnosis appropriately (12). What is more, some cases of GLM combined with breast cancer $(13,14)$. Therefore, a pathological diagnosis is the gold standard of GLM and must be obtained before the systemic treatments.

\section{The etiology of GLM remains unclear and diversity}

More and more research papers about GLM have been published in the past 5 years, which demonstrated that GLM has become common disease from a rare clinical condition. So far, the etiology of GLM is still unknown. According to these papers, the causes are currently considered as follows: (I) autoimmune disorders is a cause with more supports. Our researcher team made a comprehensive report about immunology study on nonlactating mastitis in the year of 2014 (15). Konan reported that glucocorticoids and immunosuppressive agents (azathioprine, methotrexate) were effective drugs for GLM, and glucocorticoids were more likely to be reduced while combination with immunosuppressive agents (16). Garraud et al. (17) reported that the condition of GLM went worse obviously and had a relapse after the withdrawal of antiTNF drugs. He also found some patients accompanying with rare symptoms such as lower extremity erythemas, some of which had an increased level of serum anti-nuclear antibody. Allen et al. (18) reported that GLM may be correlated with IgG4-related disease. Our study failed in a

Table 2 Composition ratio analysis of GLM therapy approaches

\begin{tabular}{|c|c|c|}
\hline Specific therapy approaches & No. of patients $(n=178)$ & $\%$ \\
\hline (2) Chinese medicine + regional wet compress + antibiotics + puncture or/and drainage & 8 & 4.5 \\
\hline (3) Chinese medicine + regional wet compress + puncture or/and drainage & 4 & 2.3 \\
\hline (4) Chinese medicine + regional wet compress + corticosteroid $+/$ - puncture or/and drainage & 2 & 1.1 \\
\hline
\end{tabular}

(1) : surgery therapy; (2) - (4): conservative therapy. 
Table 3 Analysis on general condition, therapy and recurrence

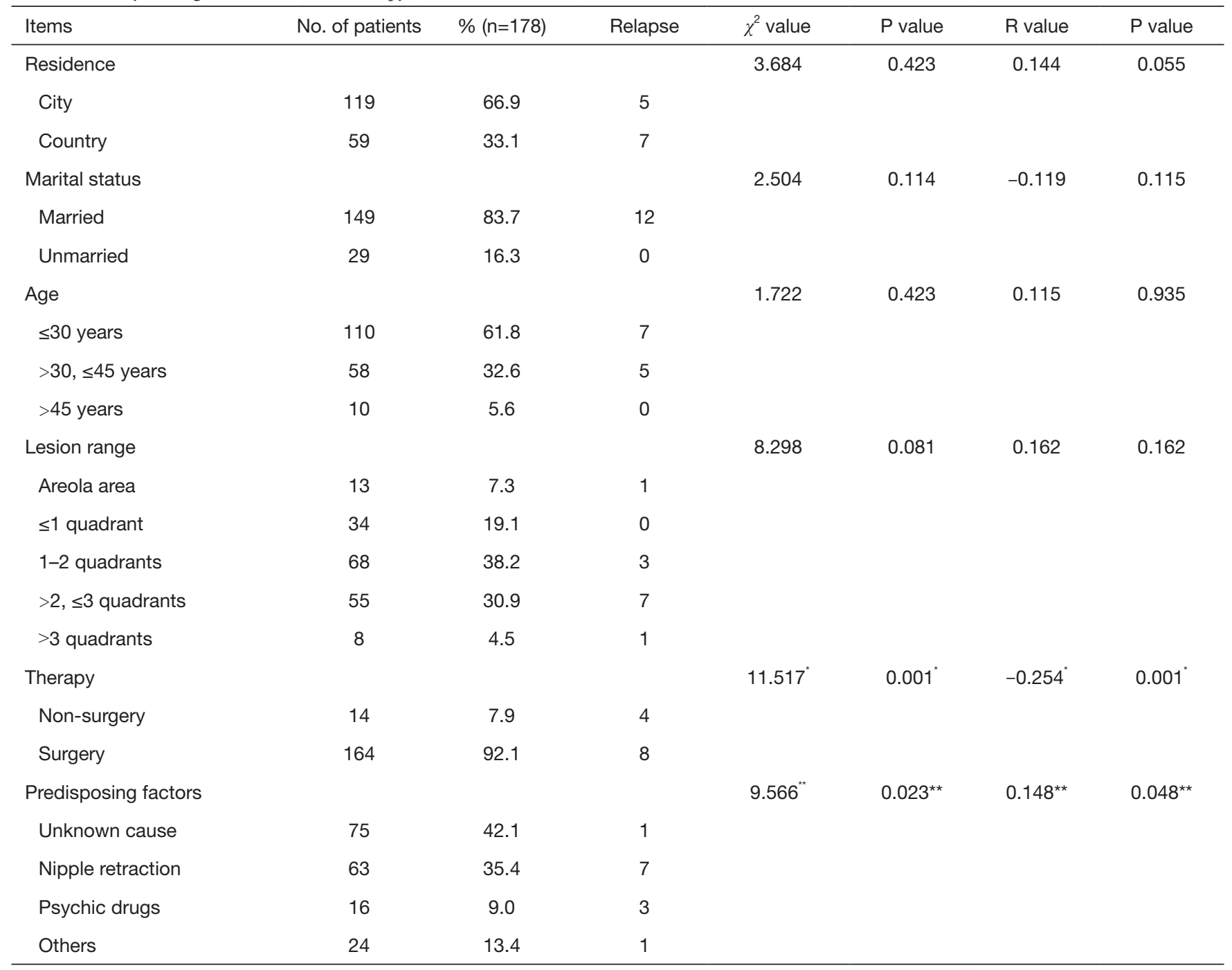

${ }^{*}$, analysis results of a combination of non-surgery therapy in small sample. Surgery therapy refers to a comprehensive treatment based on "LE + BR", and non-surgery therapy refers to other treatments included in this study; **, analysis results of combined causes in small sample. Other causes included no breastfeeding, steroid hormones, hyperprolactinemia, trauma.

further analysis on immunological causes of GLM, because only 2 cases received methylprednisolone tablets; (II) drugs medication may be another cause of GLM, such as steroid hormones and antipsychotic drugs. Eight patients in our study took contraceptives or ovulation induction drugs for more than 2 years, 16 patients took antidepressants for more than 1 year. Altintoprak et al. (19) found a similar reason about the etiology of GLM; (III) rare bacterial infections may the cause of GLM. Many reports $(2,20,21)$ claimed that the infection of coryne form bacteria or mycobacteria had some relationship with GLM, which also presented a reason for the efficacy of antibiotics or anti- tuberculosis treatment of GLM. Our study failed to find any bacteria by culture, which may be related to the culture method; (IV) nipple retraction may be a possible factor underlying the pathogenesis in GLM, which have been rarely reported on papers before. In our study, 63 patients (35.4\%) suffered from nipple retraction, accounting for the largest proportion of causes. Nipple retraction may lead to the obstruction in the lactiferous ducts of breasts and then may cause inflammation of breasts; (V) the rare causes of GLM may include hyperprolactinemia and trauma. Ten patients had no breastfeeding history; two patients suffered from hyperprolactinemia; four cases experienced 


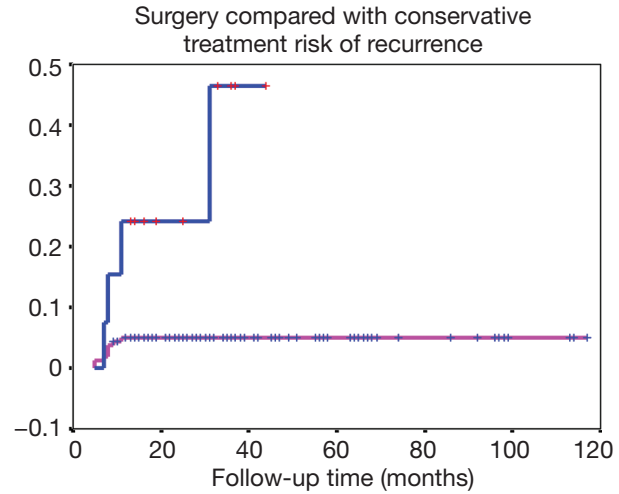

Figure 9 The Kaplan-Meier survival curve of the two types of treatment.

breast trauma. The causes above were also reported in the research of Altintoprak (19) and Nikolaev (22); (VI) gene polymorphism and gene mutation were also proven the causes of GLM in some reports. Destek (23) put forward that gene polymorphism may play an important role in the pathogenesis of GLM by detecting genes of MTHFRC667TT, PAI-1 and ACE. And Bercot (24) reported that NOD2 gene mutations was the main cause of GLM, which caused neutrophil dysfunction.

\section{In China traditional Chinese medicine may bold advantages in the non-surgical treatment of GLM}

It is relatively easy for clinicians to treat the diseases which have a clear etiology or a large number of randomized controlled trials. For example, antibiotic treatment is the key to cure acute suppurative mastitis usually caused by Gram-positive bacteria, and clinical guidelines for breast cancer are made from a large number of proofs from evidence-based medicine. Therefore, the clinicians can appropriately treat the two categories of diseases above following the guidelines or specific clinical evidences. Whereas no treatment guidelines for GLM have been formulated, because the etiology of GLM and proofs from evidence-based medicine were still unclear. Clinicians in different countries have different treatments and efficacy standards. In China, Chinese medicine theory, stemmed from macroscopic scale, emphasizes on Yin-yang balance and dialectic treatment. It is different from Western medicine, which focused on the etiology of diseases from microscopic scale. In China, Chinese medicine may have some advantages over the Western medicine on treatments of GLM, due to its unique perspective, price and patients' willing. Just as traditional herbal medicine in many other countries, Chinese medicine also obtain satisfactory outcome on the treatment of early stage of inflammatory diseases with unknown causes, especially when the price is count in Xihuang capsules, as a brand of traditional Chinese medicine, is mainly made of four kinds of common raw traditional Chinese medicines, including artificial bezoar, artificial musk, myrrh, and frankincense. It has the functions of detoxification, detumescence and abirritation for ulcers, multiple abscess, lymphadenitis, cold abscess. Therefore, our team used Xihuang capsules against GLM in a comprehensive therapy. In surgery group, we routinely preferred to use Xihuang capsules for a preoperative and postoperative treatment. In non-surgery group, patients adhered to the traditional Chinese medicine and chose to have drainages through puncture or incisions while complicated with abscess. All of them took the traditional Chinese medicine for 4 to 6 months both in surgery and non-surgery group, but the efficacy evaluation was no affected. During the time of the conservative therapy, no abnormal changes of blood routine, liver function and renal function tests were found.

\section{Surgery determines a quick recovery and a good cure rate}

In this study, 164 patients (92.1\%) underwent surgeries of lesion excision according to their willing and choice. The surgeries were carried out strictly in the whole procedures including preoperative investigations, preoperative conservative treatment, timing and modes of operation. Preoperative investigations, involving breast palpation, ultrasound examinations and MRI, carried out at the initial visit, in the middle of procedure and at the last visit which was 1 week before lesion excision operations. The purpose of these investigations was to appropriate assess the lesion range and the efficacy of conservative therapy, and then to make a correct judgment for breast volume which had to be removed in surgical therapy and the volume of the fascia flap which used to replace the lesion for a good postoperative shape for ipsilateral breast. Although MRI findings were not pathognomonic for GLM (4), MRI played a very important and unique role in calculation of lesionvolume in the procedure of GLM, and had been accepted as a routine exam at the initial visit and at last visit before lesion-excision surgeries. The same methods were optically used for postoperative assessment.

A right time of surgery is the key to a successful 


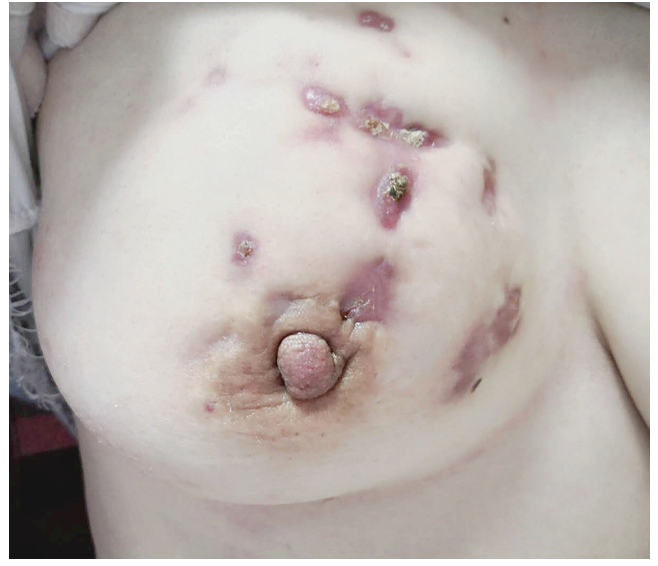

Figure 10 Clinical presentation for a 27-year-old woman with GLM at the first visit in our clinic center. The patient underwent 4 times open incision for pus drainage, due to the breast mass with abscess for 3 months. GLM, granulomatous lobular mastitis.

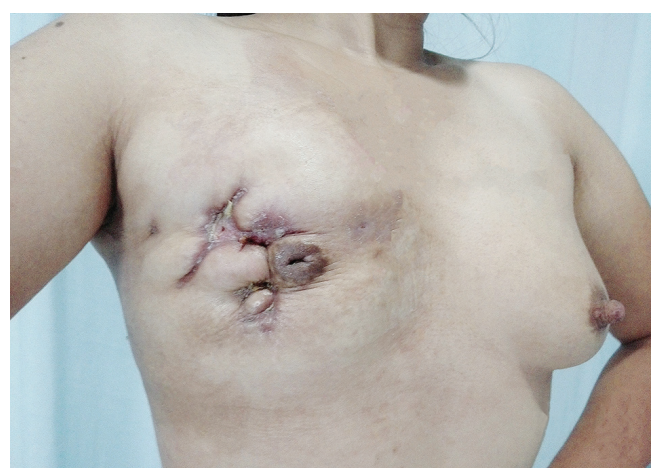

Figure 11 Clinical presentation for a 36-year-old woman with GLM at the first visit in our clinic center. The patient underwent 6 small incisions draining pus due to the right breast mass with abscess for 6 months. GLM, granulomatous lobular mastitis.

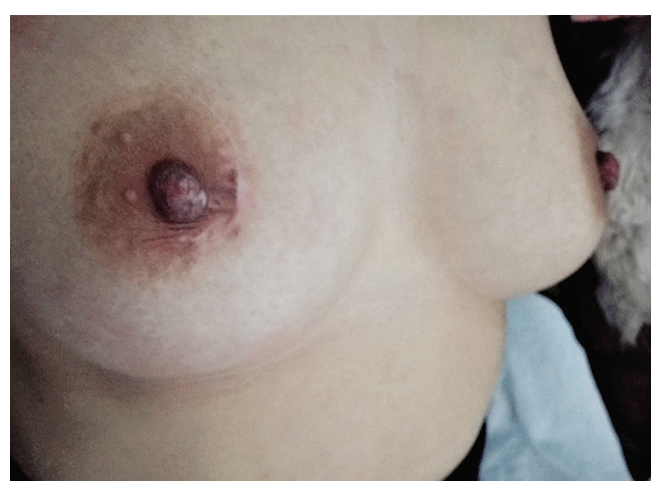

Figure 12 Two years after surgery (LE + BR) for a 26-year-old woman. The symmetry of the breast was good. treatment of GLM. As soon as the range of lesion area shrank to a size of less than 1 quadrant, the patients was informed that they could choose to have a lesion-removal surgery or continue conservative therapy. The patients were also informed the specific advantages, disadvantages and possible prognosis of both treatments at the same time. Then the patients and their families, along with their physicians decided to undergo a surgery or not. Up to $92.1 \%$ cases in this study chose to receive surgery after experiencing tortures of GLM for neatly months. Based on our study, we found the timing when the lesion became smaller than 1 quadrant is appropriate operative timing for lesion excision. Although some patients may lose a breast volume of 1 quadrant, they are basically satisfied with the appearance and shape of ipsilateral breast postoperatively.

If GLM is not combined with breast cancer, it is indeed not life-threatening. But in China, there have been a large number of GLM patients in recent years. Yet there are no institutions for epidemiological investigations. This research center is the largest provincial general hospital in Hunan Province. Before 2009, there were fewer than 10 GLM patients treated each year. The number of GLM patients admitted and treated in the past 5 years has risen rapidly. The number of patients per year has exceeded 200, showing a rapid upward trend. This phenomenon also occurs in other large general hospitals and various provinces in China. The research center is working with a number of large general hospitals in Hunan Province to investigate possible causes. In the past, in China, GLM is a rare and rare disease. It is not only basic hospitals, but also breast surgery in large hospitals. It is not known clearly about these diseases, and it is often treated as simple mastitis. If there is an abscess in the breast, the pus will be cut open. After an incision of pus, combined with antibiotics and topical dressings, the lesion may be relieved. But after a few weeks or months, an abscess is seen in another location. If the doctor takes another incision and drains the pus, it will last for several months. In a few years, the patient's breasts are scarred (see Figure 10), and even the last case of total breast resection due to necrosis of the entire breast is also present (Figure 11). It can be seen that if this disease is not controlled in time, not only is the breast damaged, but also creates physical and mental health and economic burdens on the patient.

Based on that, the research center tried to find a suitable surgical timing and surgical methods to improve the cure rate, reduce the recurrence rate and breast damage. If a GLM patient treated in timely, the appearance of the breast is very satisfactory (Figures 12-14). 


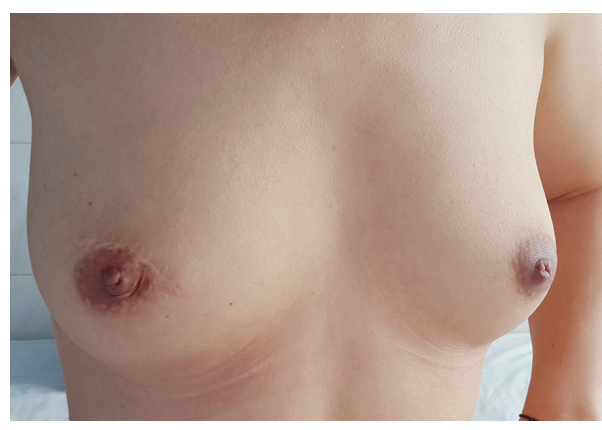

Figure 13 Three and a half years after the lesion excision combined with stage I breast reconstruction with intraglandular flap and fascia flap for a 32-year-old woman diagnosed with GLM. The appearance of both breasts was with good symmetry. GLM, granulomatous lobular mastitis.

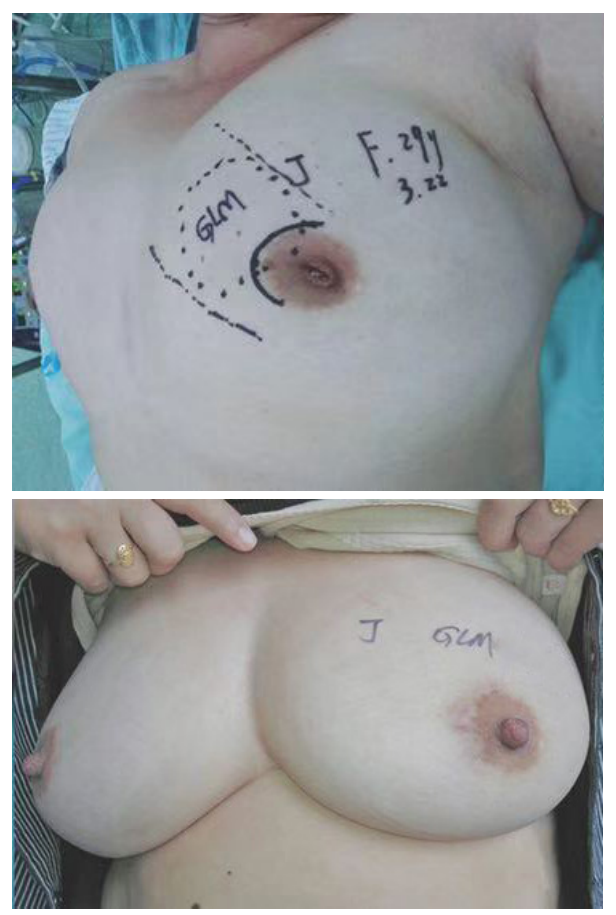

Figure 14 A 29-year-old female with a mass in upper inner quadrant of left breast (A). Seven months after the lesion excision combined with stage I breast reconstruction with intraglandular flap and fascia flap (B).

In summary, for the cases of GLM, lesion excision and stage I breast reconstruction with intraglandular flap and fascia flap (LS + BR) is an effective, safe therapy with respect to better cosmetic results, shorter recovery time, lower recurrence rate. Appropriate timing and mode of surgery can produce a high cure rate. The conservative therapy including traditional Chinese medicine, antibiotics, regional wet compress, and pus drainage provide a useful way to sufficiently control an acute inflammation preoperatively. These surgery approach and conservative therapy are therefore promising treatments, and deserve further clinical studies.

\section{Acknowledgments}

We would like to thank the patients that participated in this study.

Funding: This study was supported by Key Projects of China Hunan Provincial Science \& Technology Department (No. 2017SK2142).

\section{Footnote}

Conflicts of Interest: The authors have no conflicts of interest to declare.

Ethical Statement: The authors are accountable for all aspects of the work in ensuring that questions related to the accuracy or integrity of any part of the work are appropriately investigated and resolved. Ethical clearance was obtained from the Hunan Provincial People's Hospital Medical Faculty ethics committee (No. 201614).

\section{References}

1. Hovanessian Larsen LJ, Peyvandi B, Klipfel N, et al. Granulomatous lobular mastitis: imaging, diagnosis, and treatment. AJR Am J Roentgenol 2009;193:574-81.

2. Kamyab A. Granulomatous lobular mastitis secondary to Mycobacterium fortuitum. World J Clin Cases 2016;4:409-12.

3. Salehi M, Salehi M, Kalbasi N, et al. Corticosteroid and Azithromycin in Idiopathic Granulomatous Mastitis. Adv Biomed Res 2017;6:8.

4. Korkut E, Akcay MN, Karadeniz E, et al. Granulomatous Mastitis: A Ten-Year Experience at a University Hospital. Eurasian J Med 2015;47:165-73.

5. Zhang C, Fan P, Yu H, et al. Surgical Treatment of Idiopathic Granulomatous Mastitis( 8 cases report). Chinese Journal of General Surgery 2009;18:1216-9.

6. Zhu Y, Fan P, Zhang C. Treatment Progress of Granulomatous Lobular Mastitis. Medicine \& Philosophy(B) 2016;37:56-9. 
7. Xiao Q, Zhang C. Modern Research Progress of Non lactation Mastitis. Medicine \& Philosophy 2013;34:8-13.

8. Aghajanzadeh M, Hassanzadeh R, Alizadeh Sefat S, et al. Granulomatous mastitis: Presentations, diagnosis, treatment and outcome in 206 patients from the north of Iran. Breast 2015;24:456-60.

9. Sheybani F, Sarvghad M, Naderi HR, et al. Treatment for and clinical characteristics of granulomatous mastitis. Obstet Gynecol 2015;125:801-7.

10. Kessler E, Wolloch Y. Granulomatous mastitis: a lesion clinically simulating carcinoma. Am J Clin Pathol 1972;58:642-6.

11. Zhang C, Fan P, Liu P, et al. Applicable value of dynamic Magnetic Resonance Imaging in the Surgical Evaluation of Granulomatous Mastitis. Chinese Journal of Modern Medicine 2012;22:86-9.

12. Güven HE, Pak I, Oral S. Granulomatous mastitis: surgical outcomes. J Coll Physicians Surg Pak 2006;16:431-3.

13. Cornejo-Juárez P, Vilar-Compte D, Perez-Jimenez C, et al. Idiopathic granulomatous mastitis. Experience at a cancer center. Rev Invest Clin 2014;66:393-8.

14. Limaiem F, Khadhar A, Hassan F, et al. Coexistence of lobular granulomatous mastitis and ductal carcinoma: a fortuitous association? Pathologica 2013;105:357-60.

15. Zhang C, Kong C. Immunological studies progress of Non-lactating mastitis. Journal of Dalian Medical University 2014;36:307-13.

16. Konan A, Kalyoncu U, Dogan I, et al. Combined longterm steroid and immunosuppressive treatment regimen in

Cite this article as: Zhang C, Lei S, Kong C, Tan Y, Dai X, He J, Xiao Q, Huang S. Clinical study on surgical treatment of granulomatous lobular mastitis. Gland Surg 2019;8(6):712-722. doi: $10.21037 /$ gs.2019.11.12 granulomatous mastitis. Breast Care (Basel) 2012;7:297-301.

17. Garraud T, Barbarot S, Berthelot JM, et al. Occurrence of a granulomatous mastitis and aseptic osteitis after antiTNF therapy in a patient with pustular psoriasis. Joint Bone Spine 2016;83:107-8.

18. Allen SG, Soliman AS, Toy K, et al. Chronic Mastitis in Egypt and Morocco: Differentiating between Idiopathic Granulomatous Mastitis and IgG4-Related Disease. Breast J 2016;22:501-9.

19. Altintoprak F, Kivilcim T, Ozkan OV. Aetiology of idiopathic granulomatous mastitis. World J Clin Cases 2014;2:852-8.

20. Yu HJ, Deng H, Ma J, et al. Clinical metagenomic analysis of bacterial communities in breast abscesses of granulomatous mastitis. Int J Infect Dis 2016;53:30-3.

21. Helal TE, Shash LS, Saad El-Din SA, et al. Idiopathic Granulomatous Mastitis: Cytologic and Histologic Study of 65 Egyptian Patients. Acta Cytol 2016;60:438-44.

22. Nikolaev A, Blake CN, Carlson DL. Association between Hyperprolactinemia and Granulomatous Mastitis. Breast J 2016;22:224-31.

23. Destek S, Gul VO, Ahioglu S. A variety of gene polymorphisms associated with idiopathic granulomatous mastitis. J Surg Case Rep 2016. doi: 10.1093/jscr/rjw156.

24. Bercot B, Kannengiesser C, Oudin C, et al. First description of NOD2 variant associated with defective neutrophil responses in a woman with granulomatous mastitis related to corynebacteria. J Clin Microbiol 2009;47:3034-7. 